\title{
Decreased pulmonary distensibility in fibrosing alveolitis and its relation to decreased lung volume
}

\author{
MICHAEL J THOMPSON, H JOHN H COLEBATCH
}

\author{
From the Department of Respiratory Medicine, University of New South Wales, Prince Henry Hospital, Little \\ Bay, New South Wales, Australia
}

\begin{abstract}
The relation between pulmonary distensibility, lung volume, and elastic recoil pressure was examined in 45 patients (38 men) with cryptogenic fibrosing alveolitis (mean age 61 (SD 14) years). Exponential analysis of static pressure-volume data obtained during deflation of the lungs gave the exponent $\mathbf{K}$, an index of the distensibility of the remaining inflatable lung tissue. Total lung capacity (TLC) was measured in a body plethysmograph or by nitrogen washout. The results were compared with values obtained in 147 healthy subjects (95 men), of mean age 39 (SD 16) years. Fibrosing alveolitis decreased $\mathrm{K}$ by 0.62 (SEM 0.04$) \mathrm{kPa}^{-1}$. This decrease was approximately equal to $2 \mathrm{SD}$ of the regression of $\log \mathrm{K}$ on age in healthy subjects. TLC was decreased to a mean of $70 \%$ (SD 14\%) predicted in the patients with fibrosing alveolitis. Lung recoil pressure at maximum inspiration was about twice the expected value and regression analysis showed that most of this increase was related to the decreased $\mathrm{K}$ rather than to the decreased TLC. In the men with fibrosing alveolitis the regression of height standardised TLC $\left(\mathrm{TLC} / \mathrm{Ht}^{3}\right)$ on $\mathrm{K}$ was significant $(\mathrm{p}<0.02)$; the regression slope was similar to that for 95 healthy men, but was displaced to a smaller lung volume. The dependence of $\mathrm{TLC} / \mathrm{Ht}^{3}$ on $\mathrm{K}$ is consistent with the close relation between $\mathrm{K}$ and peripheral airspace size found in normal lungs. In fibrosing alveolitis decreased pulmonary distensibility probably reflects a decrease in airspace size, whereas most of the decrease in lung volume reflects the loss of inflatable tissue in the fibrotic process.
\end{abstract}

\section{Introduction}

Fibrosing alveolitis is a disease characterised by inflammation of lung tissue beyond the terminal bronchiole $^{12}$ with thickening of the alveolar walls by both a cellular infiltrate and a variable degree of fibrosis. Transfer factor for carbon monoxide (TLCO) is decreased,$^{34}$ but the value correlates poorly with the severity of the disease process determined by histopathology. ${ }^{15}$ Total lung capacity (TLC) and its subdivisions are also decreased, in association with decreased pulmonary compliance and increased maximum transpulmonary pressure (Plmi) ${ }^{34}$ These latter changes have been regarded as indicating an increase in lung stiffness; but, as Gibson and Pride ${ }^{6}$ have suggested, they may reflect only the consequences of a loss of inflatable tissue.

To clarify this issue the distensibility of the remaining inflatable lung tissue must be assessed independently of change in absolute lung volume or of maximum

Address for reprints requests: Dr H J H Colebatch, Department of Medicine, University of New South Wales, Prince Henry Hospital, Little Bay, NSW 2036, Australia.

Accepted 9 June 1989 transpulmonary pressure. ${ }^{7}$ Exponential analysis of static pressure-volume (PV) data obtained during deflation of the lungs resolves this problem. ${ }^{78}$ The exponent, $\mathbf{K}$, reflects the relative changes in pressure and volume and is therefore not influenced by a change in the absolute values of either measurement. $\mathrm{K}$ provides an index of pulmonary distensibility that is independent of lung size and independent of sex in healthy subjects. ${ }^{9}$ It relates directly to a morphometric estimate (mean linear intercept, $\mathrm{Lm}$ ) of the average size of airspaces in excised human lungs ${ }^{10}$ as well as the lungs of several other mammals." This relation between $\mathrm{K}$ and airspace size has been explained by the influence of airspace on surface tension forces in air inflated lungs." As airspace size decreases the magnitude of the surface component of recoil pressure increases and distensibility decreases.

Previous studies of $K$ in patients with fibrosing alveolitis have shown that $\mathrm{K}$ is decreased in some subjects. $^{712}$ The larger number of subjects in the present study has enabled us to clarify the alterations in pulmonary mechanical behaviour in fibrosing alveolitis by analysing the relation between change in lung volume, elastic recoil pressure, and distensibility. Our findings suggest that the decreased values for $\mathrm{K}$ in 
fibrosing alveolitis reflect a decrease in the size of airspaces, as in the case for normal lungs.

\section{Methods}

In 45 patients ( 38 men, seven women) a diagnosis of cryptogenic fibrosing alveolitis was made on conventional clinical and radiological criteria. ${ }^{13}$ They had noticed dyspnoea for a mean of three (range 0.1-10) years before the studies reported here were performed. In 25 of the patients the diagnosis was confirmed by histological demonstration of fibrosis of alveolar walls with varying amounts of interstitial and intra-alveolar infiltrate in lung biopsy specimens (17 patients) or at necropsy (eight patients). Thirty one patients had smoked cigarettes or were current cigarette smokers (30 men) and 11 patients were non-smokers (five men, six women); a smoking history was not available in three patients.

Total lung capacity (TLC) and maximum expiratory flow-volume (MEFV) curves were obtained by body plethysmography except for early studies on 13 patients, in which TLC was measured by prolonged nitrogen washout and forced expired volumes were obtained with a nine litre spirometer (WE Collins, Massachusetts). BTPS values were used for all lung volumes. Transfer factor for carbon monoxide (TLCO) was measured in 24 men and four women ( 25 cigarette smokers) by a single breath method using an automated system (Resparameter M4, PK Morgan) with a breath holding time of 10 seconds, an inspired carbon monoxide concentration of $0.4 \%$, and neon as the inert gas for measuring alveolar volume. Gas concentrations were measured with a gas chromatograph (Varian model 2720) and a chart recorder with a microprocessor (Varian CSD 101) to determine area, with helium as the carrier gas. Predicted values were obtained from Cotes. ${ }^{14}$

Static pressure-volume data were generated during several interrupted deflations from TLC with a computerised measurement system. ${ }^{15}$ Transpulmonary pressure $(\mathrm{PL})$ was measured with an oesophageal balloon (length $10 \mathrm{~cm}$, gas volume $0.5-1 \mathrm{ml}$ ) and a Statham differential strain gauge. The change in lung volume from TLC was obtained by electrical integration of flow at the mouth with correction for gas compression near TLC but not at lower volumes.' To assess repeatability of the pressure-volume data, after each deflation data points were displayed on a graphics terminal (Tektronix 4006). An exponential function of the form

$$
V=\mathrm{A}-\mathrm{Be}^{-\mathrm{K} P}
$$

(where $V$ is lung volume and $P$ is static recoil pressure and $A, B$, and $K$ are constants) was fitted to the pressure-volume data from TLC to a lower volume limit not less than $50 \%$ of TLC (mean $60 \%$ (SD 6\%)
TLC) ${ }^{8}$ The lower volume limit was also influenced by the increase in $\mathrm{RV} / \mathrm{TLC} \%$ (mean $43 \%$, range 27 $65 \%)$. The pressure-volume data and the fitted exponential function were displayed on the graphics terminal.

The exponential constant $\mathrm{K}$ describes the shape of the pressure-volume curve independently of TLC. The constant $A$ is the volume asymptote and $B$ is the difference between $\mathbf{A}$ and the volume at zero recoil pressure. The distribution of the original pressurevolume data about the derived curve was quantified by the ratio of residual variance to the total variance for volume. Mean residual variance was $1.2 \%$ (SD 0.8\%). The ratio $\mathrm{A} / \mathrm{TLC} \%$ is a guide to the fit of the curve to data points near TLC. The mean value was $101.8 \%$ (SD 2.6\%); in only three subjects (one man, two women) did the value exceed $105 \%$ and in these cases it reflected the trend of the data points.

PL during a sustained maximum inspiratory effort at full inflation, maintained for about one second (PLmi), was measured directly and was the mean of the four highest values. Static recoil pressure at $90 \%$ TLC $\left(\mathrm{PL}_{9_{0}}\right)$ was derived from the exponential function.

For lung volumes and elastic behaviour comparisons were made with values obtained for 147 healthy non-smokers (95 men, 52 women), mean age 39 (SD 16) years, who were studied by methods similar to those described above. ${ }^{9} \mathrm{~K}$ has a log-normal distribution with respect to age in healthy subjects; for this reason we made the comparisons using $\ln \mathrm{K}$ where relevant. Linear regression, multiple regression, covariance analysis of linear regression, $R$, and Student's $t$ test were performed as described by Snedecor. ${ }^{16}$

\section{Results}

Anthropometric data at the time of study are given in table 1 . The mean duration of dyspnoea was $3 \cdot 2$ (range $0 \cdot 1-10)$ years. Subjects in the smoking group had smoked an average of 28 (SD 14, range 10-60) cigarettes a day for an average of 37 (SD 14, range 1267) years.

Lung volumes were decreased and the decrease was greater in the women than in the men, but this difference did not reach significance $(p>0.05$; table 1). Owing to the relatively greater decrease in TLC than in RV the ratio RV/TLC\% was increased. This increase was significant only in the men, in whom it was $15 \%$ (SD $30 \%$ ) greater than the predicted value $(p<0.01) . F E V_{1}$ was decreased but its ratio to the decreased FVC was similar to that obtained in healthy subjects. TLCo was decreased (mean $42 \%$, SD $18 \%$ predicted); in the three non-smokers the value averaged $44 \%$ of predicted. In the men multiple linear regression analysis of TLCO on TLC and 1/K showed 
Table 1 Anthropometric data, lung volumes, and ventilatory function in fibrosing alveolitis (means with standard deviations in parentheses and percentages of predicted values in square brackets)

\begin{tabular}{lcc}
\hline & Men $(n=38)$ & Women $(n=7)$ \\
\hline Age (y) & $62(12)$ & $56(22)$ \\
Height (cm) & $172(8)$ & $161(4)$ \\
Weight (kg) & $72(12)$ & $59(9)$ \\
TLC(l) & $4.93(1.24)$ & $3.31(0.78)$ \\
& {$[71.3(14)]$} & {$[60.6(14)]$} \\
FRC(l) & $3.09(0.85)$ & $2.25(0.64)$ \\
& {$[80.1(18.9)]$} & {$[68.9(19.2)]$} \\
RV(l) & $2.02(0.58)$ & $1.60(0.27)$ \\
VC(l) & {$[80.6(26.0)]$} & {$[70.0(11.7)]$} \\
& $2.92(0.94)$ & $1.7(0.62)$ \\
FEV $(1)$ & {$[66.7(17.3)]$} & {$[55.0(22.8)]$} \\
& $2.19(0.68)$ & $1.33(0.43)$ \\
FEV,$/ F V C(\%)$ & {$[73.5(17.9)]$} & {$[58.7(25.4)]$} \\
& $76.2(10.3)$ & $79.3(6.7)$ \\
& {$[103.6(14.9)]$} & {$[100.2(8.8)]$} \\
\hline
\end{tabular}

Lung volumes are decreased $(p<0.01)$ and the decrease did not differ significantly in men and women. The ratio $\mathrm{FEV}_{1} / \mathrm{FVC}$ is similar to the value for healthy subjects.

TLC - total lung capacity; FRC - functional residual capacity; RV-residual volume; VC - vital capacity; FEV _-forced expiratory volume in one second.

no significant relation between TLCO and TLC or between TLCO and $1 / \mathrm{K}$ ( $\mathrm{p}>0.4$ and $\mathrm{p}>0.5$ respectively).

An example of the pressure-volume data with a fitted single exponential function is shown in figure 1 . $\mathrm{K}$ and $\ln \mathrm{K}$ were decreased (table 2 , fig 2 ) and the decrease did not differ significantly in men and women. In 25 subjects $\mathrm{K}$ was less than the lower limit of the $95 \%$ confidence interval in healthy subjects for the regression of $\ln \mathrm{K}$ on age. $\mathrm{K}$ was slightly higher in the 31 smokers (mean (SD) $0.97(0.35) \mathrm{kPa}^{-1}$ ) than in the 11 non-smokers $\left(0.86,(0 \cdot 28) \mathrm{kPa}^{-1}\right)$, but the
Table 2 Exponential analysis of static pressure-volume data for 45 patients with fibrosing alveolitis (means with standard deviations in parentheses and percentages of predicted values in square brackets except for $\ln K$ )

\begin{tabular}{|c|c|c|}
\hline & $\operatorname{Men}(n=38)$ & Women $(n=7)$ \\
\hline $\mathrm{K}\left(\mathrm{kPa}^{1} \times 10\right)$ & $9.71(3 \cdot 15)$ & $7.99(3.21)$ \\
\hline $\begin{array}{l}\text { In } \underset{\text { Kredicted }}{\mathrm{K}\left(\mathrm{kPa}^{\prime}{ }^{\prime}\right)} \\
\text { Prmi (kPa) }\end{array}$ & $\begin{array}{c}{[(62.1+(20.3)]} \\
-0.073+(0.29) \\
0.419(0.200) \\
5.8(2.45) \\
{[(203.3+(93.1)]}\end{array}$ & $\begin{array}{c}{[(51.3+(16.5)]} \\
-0.302+(0.44) \\
0.395(0.200) \\
4.81(1.21) \\
{[(208.1 *(70)]}\end{array}$ \\
\hline $\mathrm{PL}_{90}(\mathrm{kPa})$ & $\begin{array}{c}1.81(0 \cdot 5) \\
{[(151 \cdot 2 \dagger(49 \cdot 4)]}\end{array}$ & $\begin{array}{c}1.90(0.5) \\
{\left[\left(158.6^{*}(24.5)\right]\right.}\end{array}$ \\
\hline
\end{tabular}

$\mathrm{K}$-the index of lung distensibility; Ptmi-static recoil pressure at maximal inspiration; $\mathrm{PL}_{90}$ static recoil pressure at $90 \%$ TLC ${ }^{*} p<0.05 ; \mathrm{tp}<0.01$ : significance of the difference from the predicted value for healthy subjects. The decrease in $\mathrm{K}$ and the increase in static recoil pressures were not significantly different in men and women.

difference did not reach significance $(p>0 \cdot 2)$. Six male smokers had values for $\mathrm{FEV}_{\mathrm{l}} / \mathrm{FVC}<65 \%$ (mean $59 \%$ ); their values for $\mathrm{K}$ (mean (SD) $\left.1.33(0.47) \mathrm{kPa}^{-1}\right)$ were greater than for the 11 non-smokers $(p<0.05)$. The ratios for $\mathbf{B} / \mathbf{A} \%$ in patients, both in men (mean (SD) $61 \%(15 \%))$ and in women $(55 \%(14 \%))$, were less than in healthy subjects $(p<0.005$ and $<0.05)$. This decrease reflects the decrease in TLC in both groups.

To estimate the average decrease in $\mathrm{K}$ in the 45 patients with fibrosing alveolitis, 147 healthy subjects were included in a multiple regression analysis of $\ln \mathrm{K}$ on age (y) and fibrosis (coded as 1), which gave the equation

$\ln \mathrm{K}=\left(5.86 \times 10^{-3} \times\right.$ age $)-(0.524 \times$ fibrosis $)+$ 0.0584

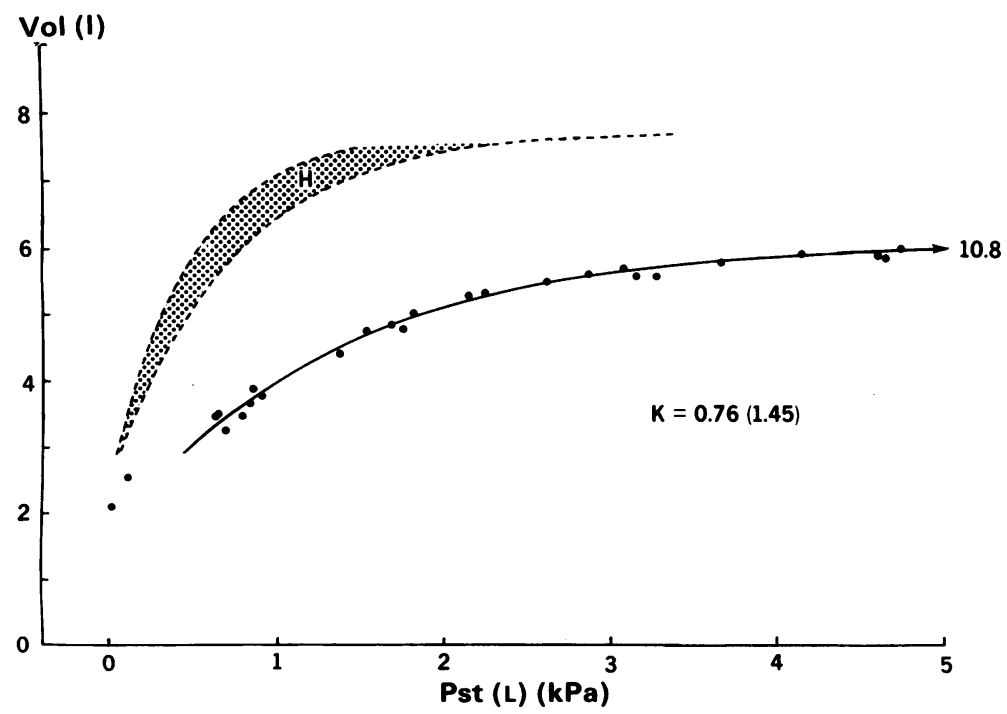

Fig 1 Static pressure (Pst (L))volume data for a 56 year old male smoker with fibrosing alveolitis. $A$ single exponential function has been fitted over the upper $49 \%$ of lung volume (residual variance $0.8 \%$ ). The shaded area is the $1 S D$ range for a healthy non-smoker at the predicted total lung capacity. The value for the exponent, $K$, was lower than the predicted value (given in parentheses). 


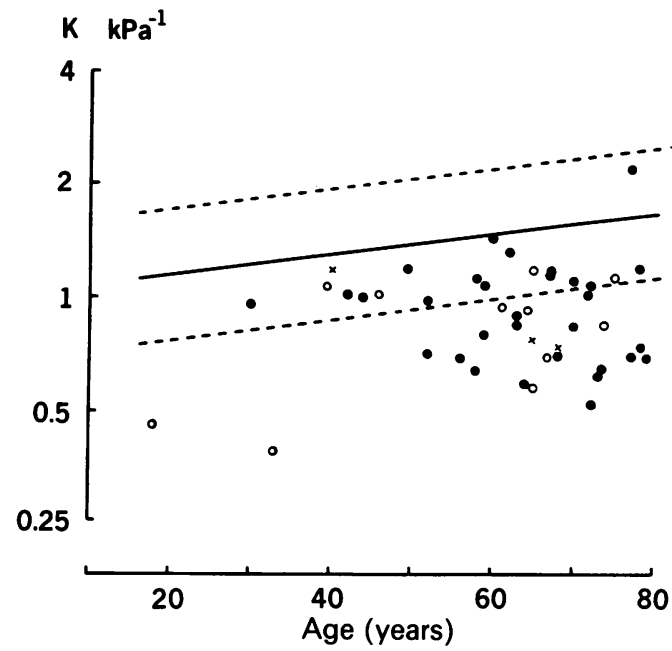

Fig 2 Values of lung distensibility ( $K$ on a logarithmic scale) for 45 patients with fibrosing alveolitis: 31 smokers (O), 11 non-smokers (O), and three subjects with no known smoking history $(x)$. The mean regression slope and $95 \%$ confidence interval are from the regression of In $K$ on age for 124 healthy subjects $(H)$.

The coefficients for age and fibrosis were significant (SEM $1.06 \times 10^{-3}$ and $4.61 \times 10^{-2} ; \mathrm{p}<0.001$ for each). The coefficient for fibrosis was more than twice the SD $(0.20$; ref 9$)$ of the regression of $\ln \mathrm{K}$ on age for healthy subjects. From equation 2 it may be shown that at age 61 years fibrosis decreased $\mathrm{K}$ by 0.62 (SEM $0.04) \mathrm{kPa}^{-1}$.

PLmi and $\mathrm{PL}_{90}$ were increased (table 2). In the men we examined whether, after allowance had been made for the decreased values for K, PLmi was related to the decreased lung volume in fibrosis. In 95 healthy men the regression of PLmi on $1 / \mathrm{K}$ (both in $\mathrm{kPa}$ ) was given by

$$
\text { Plmi }=3.70 \times 1 / \mathrm{K}+0.84
$$

and in the 38 men with fibrosing alveolitis by

$$
\text { Plmi }=4.83 \times 1 / K+0.39 .
$$

The regression coefficients were significant $(R=0.53$ and $0.61 ; p<0.001$ in each case). Covariance analysis showed that the regression slopes were similar and the difference in elevation did not reach significance $(p>0.05)$. The values were therefore pooled and the regression of PLmi on 1/K for 133 men (fig 3) was given by

$$
\text { Plmi }=4.88 \times 1 / \mathrm{K}+0.029 .
$$

For this combined group a multiple regression of PLmi on $1 / \mathrm{K}$ and lung volume-expressed either as TLC\% predicted or standardised for height as TLC/ $\mathrm{Ht}^{3}$ showed that the coefficient for lung volume was not significant in either case $(p>0.5 ; F=0.004$ and $0 \cdot 01)$. This result excludes a quantitative relation

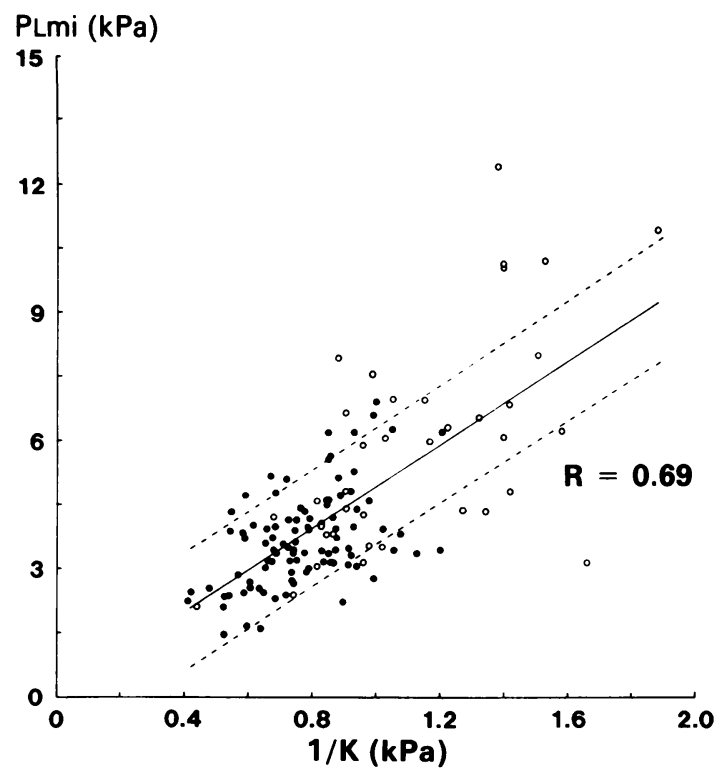

Fig 3 Regression of maximum inspiratory transpulmonary pressure (PLmi) on the inverse of lung distensibility $(1 / K)$ for 95 healthy men (O) and 38 men with fibrosing alveolitis (O): mean regression line with $1 S D$ (interrupted lines). $R$ is the correlation coefficient. The slope of regression is significant $(p<0.001)$.

$\mathrm{TLC} / \mathrm{Ht}^{3}\left(\mathrm{~L} / \mathrm{m}^{3}\right)$

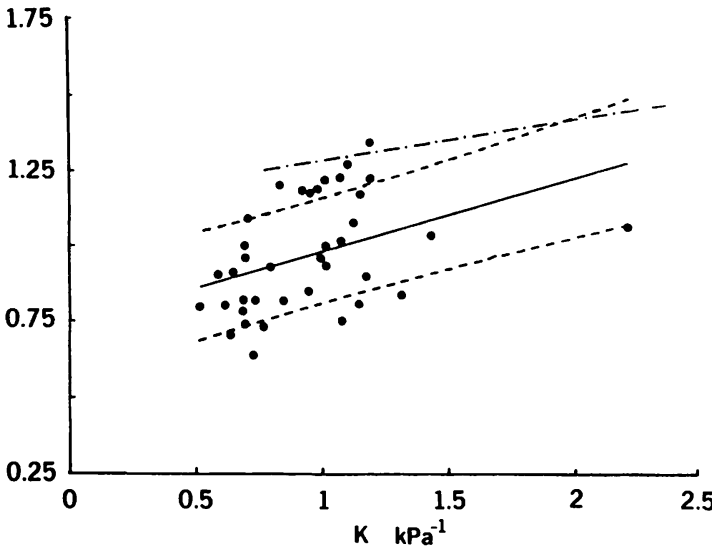

Fig 4 Regression of total lung capacity $(T L C) / \mathrm{Ht}^{3}$ on lung distensibility $(K)$ for 38 men with fibrosing alveolitis: mean regression line with $1 S D$ (interrupted lines) and mean regression (dot-dashed line) for 95 healthy male nonsmokers. The slope of regression is significant $(p<0.02)$ and similar to the slope for healthy subjects $(p>0 \cdot 1)$, but in alveolitis values for $\mathrm{TLC} / \mathrm{Ht}^{3}$ are systematically decreased $(p<0.001)$. 
between an increased PLmi and a decreased TLC. To assess a qualitative effect of decreased lung volume, however, the multiple regression was performed with fibrosis coded as 1 . This regression gave the equation Plmi $=(4.23 \times 1 / \mathrm{K})+(0.65 \times$ fibrosis $)+0.41$ (6) Both coefficients were significant $(F=61 \cdot 3$ and $4 \cdot 2)$. (The addition of an age term to the above regression analysis gave a coefficient $(-0.011$, SEM 0.008) that was non-significant; it was therefore omitted.)

In the 38 men with fibrosing alveolitis there was a significant regression of TLC standardised for height $\left(\mathrm{TLC} / \mathrm{Ht}^{3}, 1 / \mathrm{m}^{3}\right)$ on $\mathrm{K}\left(\mathrm{kPa}^{-1}\right)$, given by

$$
\text { TLC } / \mathrm{Ht}^{3}=0.237 \times \mathrm{K}+0.737
$$

The regression slope was significant $(p<0.02)$ and covariance analysis showed that the slope did not differ significantly from that for 95 healthy men, ${ }^{17}$ but the values in patients with fibrosing alveolitis were systematically decreased ( $p<0.001$; fig 4 ). When the above regression analysis was limited to the 30 men in whom TLC had been measured in a body plethysmograph a similar result was obtained.

\section{Discussion}

Measurement of pulmonary distensibility has provided important information about the mechanisms underlying the altered pulmonary mechanical behaviour in fibrosing alveolitis. The decrease in lung volume and increase in lung elastic recoil pressure in the present study are similar to findings in previous reports. ${ }^{2-5}$ Owing to its dependence on the force developed by the chest wall, however, lung elastic recoil pressure does not necessarily reflect the elastic behaviour of the remaining inflatable lung tissue. ${ }^{6}$ In healthy men the pressure that can be developed by the chest wall increases at lung volumes less than TLC. At $70 \%$ of actual TLC this increased pressure is of the order of 5-7 kPa ${ }^{18} \mathrm{~A}$ major part of the increased PLmi in men with fibrosis was therefore expected to relate to the decrease in TLC, as suggested by the model of pressure-volume behaviour in fibrosing alveolitis developed by Gibson and Pride. ${ }^{6}$ In this model PLmi increases because TLC decreases. We might therefore expect to find an inverse relation between PLmi and TLC, but no relation between PLmi and 1/K. The findings from the present analysis do not support this model. Fibrosis, presumably because of loss of inflatable units, added an increment of $0.65 \mathrm{kPa}$ to PLmi (equation 6 above), but there was no relation between the magnitude of the increase in PLmi and the decrease in TLC. PLmi increased as the lungs became less distensible.

The contribution made to PLmi by a decreased K may be estimated on the basis of equation 6 . In the men with fibrosis the mean $\mathrm{K}$ was $0.93 \mathrm{kPa}^{-1}$ (from ln K, table 2), for which the expected Plmi is
$5.61 \mathrm{kPa}$. For a healthy man aged 62 years the mean $\mathrm{K}$ is $1.54 \mathrm{kPa}^{-1}$, giving a PLmi of $3.16 \mathrm{kPa}$. In the fibrosis group therefore the increase in PLmi was $2.45 \mathrm{kPa}$. Of this increase, $0.65 \mathrm{kPa}(27 \%)$ may be related to lung shrinkage; the remainder- $1.8 \mathrm{kPa}(73 \%)$-is related to decreased $\mathrm{K}$. Clearly therefore in the men with fibrosing alveolitis most of the increase in PLmi reflected the decreased distensibility of their lungs. The additional increment from decreased TLC, or from any other source, was small.

Over a long period the expected effect of an increased inflating pressure would be to distend the remaining airspaces a little more than is normally the case. A similar process, involving stretching of alveolar tissue, appears to be responsible for an increase in TLC and in $K$ in some patients with asthma. ${ }^{19}$ Any such effects would oppose the decreases in TLC and $\mathrm{K}$ reported here.

Although there was an average decrease in $\mathrm{K}$ of a little more than 2 SD, some individuals with fibrosing alveolitis had values within the normal range. This was also the case in previous studies. ${ }^{712}$ In their study of pulmonary fibrosis Finucance and Pritchard ${ }^{12}$ found that 10 of 18 patients $(56 \%)$ had values for $\mathrm{K}$ below the normal range, a proportion similar to that found in our subjects. The use of a different normal range by Gibson et al may explain why these authors found that $\mathrm{K}$ was decreased in only two of nine subjects with fibrosis; on the basis of our normal range $K$ was decreased in six of their subjects. The effect of cigarette smoking is also relevant. Most of our patients with fibrosing alveolitis were smokers and smoking increases $\mathrm{K}$ in some subjects. ${ }^{17}$ When these smokers as a group were compared with the non-smokers with fibrosing alveolitis, smoking did not increase $\mathrm{K}$ significantly. But in six smokers with decreased $\mathrm{FEV}_{1} / \mathrm{FVC}$ ratios $\mathrm{K}$ was higher than in non-smokers. Airflow limitation has no direct effect on $\mathrm{K}$ and, in this group, the decreased ratios for $\mathrm{FEV}_{1} / \mathrm{FVC}$ are probably a marker for the effects of smoking on the lungs.

What is responsible for the decrease in distensibility in fibrosing alveolitis? A correlation between pulmonary compliance and the severity of fibrosis (as graded from biopsy tissue ${ }^{5}$ does not imply that fibrosis itself is directly responsible for decreased distensibility. In bleomycin induced pulmonary fibrosis Goldstein et $a P^{0}$ observed a dissociation between the composition of connective tissue and the pressure-volume behaviour of the lungs. In addition, collagen is inextensible $e^{21}$ and is more likely to limit the maximum volume of the lungs than to influence the relative changes in volume and transpulmonary pressure.

The increased distensibility of the lungs with aging and clinical emphysema presents a similar problem. In neither of these conditions is there any consistent 
change in the quantity of parenchymal collagen and elastin in the human lung. ${ }^{22}$ Lung distensibility (or its inverse, elasticity) is therefore unlikely to reflect the behaviour of the fibrous framework of the lungs directly. This view is supported by the finding that there is no change in the tensile strength of the human lung parenchyma with advancing age. ${ }^{23}$ But by contrast all of the increase in $\mathrm{K}$ with age may be explained by an increase in airspace size with age. ${ }^{24}$ Further evidence of the structural basis for lung distensibility is provided by the results of studies made in animals. In the excised lungs of rats, cats and dogs tissue elastic properties (as assessed in saline filled lungs) had no discernible effect on the distensibility of air inflated lungs." Distensibility was closely related to the average size of peripheral airspaces, which accounted for more than $80 \%$ of the variance of $K$.

The relation between TLC and K helps to elucidate the meaning of a change in $K$ in terms of a change in airspace size. TLC is determined by the number and size of airspaces in the lungs. $K$ is not related to alveolar number ${ }^{11}$ or to height. ${ }^{917}$ But in intact human subjects $\mathrm{K}$ is an independent determinant of TLC, ${ }^{17} \mathrm{a}$ finding that is difficult to explain unless $\mathrm{K}$ reflects airspace size. In the men with fibrosing alveolitis a significant relation between height standardised TLC and $\mathrm{K}$ again implies that $\mathrm{K}$ is related to airspace size in these patients.

There are two ways in which airspace size may be decreased in fibrosing alveolitis. Firstly, an increase in interstitial tissue may increase parenchymal volume at the expense of airspace volume. Secondly, fibrous tissue, by imposing a limit to inflation, may be responsible for an acquired decrease in airspace size. Possibly, both processes play a part, but to different degrees in different individuals. Detailed morphometric studies of fibrotic lungs fixed in inflation are required to differentiate these possibilities.

For the reasons given above, the decreased values for $\mathrm{K}$ in fibrosing alveolitis probably reflect a decrease in airspace size. The lungs therefore become less distensible because of an increase in the density of surface forces (increase in the surface to volume ratio) and not directly because of the presence of fibrosis, though fibrosis probably determines the elastic limit. It is also evident from the systematic decrease in the height standardised TLC that in fibrosing alveolitis loss of inflatable tissue is chiefly responsible for the decreased lung volume.

In this study of patients with fibrosing alveolitis we have tried to elucidate the relationships between the changes in elastic recoil pressure, lung volume, and distensibility. The increased Plmi predominantly reflects the decreased pulmonary distensibility. The decreased lung volume is mainly a consequence of the loss of inflatable tissue. Pulmonary distensibility is decreased probably because of an accompanying $\stackrel{\overrightarrow{\vec{S}}}{\overrightarrow{7}}$ decrease in the size of peripheral airspaces caused by $\bar{T}$ the fibrosis. This analysis assists an understanding of $\frac{\bar{O}}{}$ the altered pulmonary mechanical behaviour in fibrosing alveolitis.

We thank Drs GB Field, J Schneeweiss, and B Jarvie for the opportunity to include patients under their care $\vec{\circ}$ in this study. We are indebted to the department of $\overrightarrow{\vec{A}}$ medical illustration, University of New South Wales, $\stackrel{\omega}{\rho}$ for photographing the illustrations, and to Mrs $\mathrm{N}$ Thomas for assistance in preparing the manuscript. Mr CKY Ng assisted with analysis of the data. This work was supported in part by a grant from the 0 National Health and Medical Research Council of $N$ Australia.

\section{References}

1 Scadding JG, Wilson KFW. Diffuse fibrosing alveolitis (diffuse interstitial fibrosis of the lungs). Correlation of $\frac{3}{\sigma}$ histology at biopsy with prognosis. Thorax $\stackrel{\mathbb{C}}{-}$ 1967;22:291-304.

2 Crystal RG, Fulmer JD, Roberts WC, Moss LM, et al. Idiopathic pulmonary fibrosis: clinical, histologic, radiographic, physiologic, scintigraphic, cytologic, and biochemical aspects. Ann Intern Med 1976;85:769-88.

3 Yernault JC, deJonghe $\mathbf{M}$, deCoster A, Englert $M$. Pulmonary mechanics in diffuse fibrosing alveolitis. Bull Physiopathol Respir 1975;11:231-44.

4 Murphy DMF, Hall DR, Peterson MR, Lapp NL. The effect of diffuse pulmonary fibrosis on lung mechanics. Bull Europ Physiopathol Respir 1981;17:27-41.

5 Fulmer JD, Roberts WC, von Gal ER, Crystal RG. Morphologic-physiologic correlates of the severity of fibrosis and degree of cellularity in idiopathic pulmonary fibrosis. J Clin Invest 1979;63:665-76.

6 Gibson GJ, Pride NB. Pulmonary mechanics in fibrosing alveolitis. The effects of lung shrinkage. Am Rev Respir Dis 1977;116:637-47.

7 Gibson GJ, Pride NB, Davis J, Schroter RC. Exponential description of the state pressure-volume curve of nor- O mal and diseased lungs. Am Rev Respir Dis 1979;120:799-811.

8 Colebatch HJH, Ng CKY, Nikov N. Use of an exponential function for elastic recoil. $J$ Appl Physiol 을 1979;46:387-43.

9 Colebatch HJH, Greaves IA, Ng CKY. Exponential $N$ analysis of elastic recoil and aging in healthy males and $N$ females. J Appl Physiol 1979;47:683-91

10 Greaves IA, Colebatch HJH. Elastic behaviour and $\omega$ structure of normal and emphysematous lungs post mortem. Am Rev Respir Dis 1980;121:127-36.

11 Haber PS, Colebatch HJH, Ng CKY, Greaves IA. Alveolar size as a determinant of pulmonary disten- $\stackrel{\infty}{+}$ sibility in mammalian lungs. $J$ Appl Physiol $\bigcirc$ 1983;54:837-45.

12 Finucane KE, Pritchard MG. Mechanical properties of $\mathbb{D}$ the lung in diffuse interstitial lung disease. Aust $N Z J \frac{\varrho}{\Phi}$ Med 1984;14:755-61. 
13 Turner-Warwick M, Burrows B, Johnson A. Cryptogenic fibrosing alveolitis: clinical features and their influence on survival. Thorax 1980;35:171-80.

14 Cotes JE. Lung function: assessment and application in medicine. 3rd ed. Oxford: Blackwell, 1975.

15 Colebatch HJH, Nail BS, Ng CKY. Computerised measurement of pulmonary conductance and elastic recoil. J Appl Physiol 1978;44:611-8.

16 Snedecor GM. Statistical methods. 5th ed. Ames, Iowa: Iowa State University Press, 1956.

17 Colebatch HJH, Greaves IA, Ng CKY. Pulmonary distensibility and ventilatory function in smokers. Bull Europ Physiopathol Respir 1985;21:439-47.

18 Agostini E, Hyatt RE. Static behaviour of the respiratory system. In: Macklem PT, Mead J, eds. Handbook of physiology, section 3: The respiratory system. Vol III. Maryland: American Physiological Society, 1986: 113-30.
19 Greaves IA, Colebatch HJH. Large lungs after childhood asthma: a consequence of enlarged airspace. Aust $N Z J$ Med 1985;15:427-34.

20 Goldstein RH, Lucey EC, Franzblau C, Snider GL. Failure of mechanical properties to parallel changes of lung connective tissue composition in bleomycininduced pulmonary fibrosis in hamsters. Am Rev Respir Dis 1979;120:67-73.

21 Stromberg DD, Wiederhielm CA. Viscoelastic description of a collagenous tissue in simple elongation. $J$ Appl Physiol 1969;26:857-62.

22 Hance AJ, Crystal RG. The connective tissue of the lung. Am Rev Respir Dis 1975;112:657-711.

23 Pierce JA. Tensile strength of human lung. $J$ Lab Clin Med 1965;66:652-8.

24 Colebatch $\mathrm{HJH}, \mathrm{Ng}$ CKY. A longitudinal study of pulmonary distensibility in healthy adults. Respir Physiol 1986;65:1-11. 